\title{
Repetitive transcranial magnetic stimulation over inferior frontal cortex impairs the suppression (but not expression) of action impulses during action conflict
}

\section{A. Dilene van Campen ${ }^{1,2,3}$}

K. Richard Ridderinkhof ${ }^{1,2}$

\author{
Richard Kunert $^{3,4}$ | Wery P. M. van den Wildenberg1,2
}

${ }^{1}$ Department of Psychology, University of Amsterdam, Amsterdam, The Netherlands

${ }^{2}$ The Amsterdam Brain and Cognition Center (ABC), University of Amsterdam, Amsterdam, The Netherlands

${ }^{3}$ Donders Institute for Brain, Cognition and Behaviour, Radboud University, Nijmegen, The Netherlands

${ }^{4}$ Max Planck Institut für Psycholinguistik, Nijmegen, The Netherlands

\section{Correspondence}

A. Dilene van Campen, University of Amsterdam, Nieuwe achtergracht 129-B, 1018 WT Amsterdam, The Netherlands. Email: A.D.vanCampen@gmail.com

\section{Funding information}

Netherlands Organization for Scientific Research (NWO) Open Competition grant (to K. R. R., W. P. M. v. d. W.)

\begin{abstract}
In the recent literature, the effects of noninvasive neurostimulation on cognitive functioning appear to lack consistency and replicability. We propose that such effects may be concealed unless dedicated, sensitive, and process-specific dependent measures are used. The expression and subsequent suppression of response capture are often studied using conflict tasks. Response-time distribution analyses have been argued to provide specific measures of the susceptibility to make fast impulsive response errors, as well as the proficiency of the selective suppression of these impulses. These measures of response capture and response inhibition are particularly sensitive to experimental manipulations and clinical deficiencies that are typically obfuscated in commonly used overall performance analyses. Recent work using structural and functional imaging techniques links these behavioral outcome measures to the integrity of frontostriatal networks. These studies suggest that the presupplementary motor area (pre-SMA) is linked to the susceptibility to response capture whereas the right inferior frontal cortex (rIFC) is associated with the selective suppression of action impulses. Here, we used repetitive transcranial magnetic stimulation (rTMS) to test the causal involvement of these two cortical areas in response capture and inhibition in the Simon task. Disruption of rIFC function specifically impaired selective suppression of conflicting action tendencies, whereas the anticipated increase of fast impulsive errors after perturbing pre-SMA function was not confirmed. These results provide a proof of principle of the notion that the selection of appropriate dependent measures is perhaps crucial to establish the effects of neurostimulation on specific cognitive functions.
\end{abstract}

\section{K E Y W O R D S}

action conflict, action impulse, IFC, inhibition, pre-SMA, Simon task, TMS

\section{1 | INTRODUCTION}

In recent years, we have seen an increased interest in the effects on cognitive functioning of noninvasive transcranial neurostimulation tools, such as repetitive transcranial magnetic stimulation (rTMS) and transcranial direct current stimulation (tDCS). One important aim of such studies is to causally link specific cognitive processes and behaviors to (changes in) the functioning of specific brain regions and networks in healthy human adults. Notably, extensive review studies have failed to reveal consistent and reproducible effects of tDCS on attentional functions (Reteig, Talsma, van Schouwenburg, \& Slagter, 2017), working memory (Russowsky-Brunoni \& Vanderhasselt, 2014), or executive function, language, and memory (Horvath, Forte, \& Carter, 2015). rTMS protocols seem to suffer less severely from 
such issues (e.g., Hsu, Ku, Zanto, \& Gazzaley, 2015), but unexpected or null findings are frequently reported here as well (for review, see, e.g., Guse, Falkai, \& Wobrock, 2010).

A variety of potential reasons for such a lack of robust findings has been proposed, tested, and discussed, including the intensity, duration, site, or repetition of stimulation, and various other aspects of study design. Here, we propose an additional source of inconsistency in observed cognitive effects of neurostimulation, and we present a proof-of-concept study to address that issue and assess the associated outcomes. We argue that, at least in some cases, null findings or nonreplicable findings may be due to problems with process pureness and sensitivity of the dependent measures that reflect the cognitive processes as targeted by the stimulation. If measures are not sufficiently process pure and/ or sensitive, then the subtle and complex effects of neurostimulation may not become manifest in clear and robust ways.

As a case in point, we will consider interference effects in the conflict paradigm, for which conflicting results for neurostimulation effect have been reported. For instance, inhibition of a competing response was reduced after rTMS over presupplementary motor area (pre-SMA) in a response competition task (Duque, Olivier, \& Rushworth, 2013). Interference effects were diminished after tDCS over the dorsolateral prefrontal cortex (DLPFC) in a spatial-numerical association of response codes (SNARC) task (Schroeder, Pfister, Kunde, Nuerk, \& Plewnia, 2016) and after rTMS over posterior parietal cortex but not medial frontal cortex in an Eriksen flanker task (Jin, Olk, \& Hilgetag, 2010). Response interference effects were not modulated by stimulating the DLPFC using rTMS in a Stroop task (Vanderhasselt, De Raedt, Baeken, Leyman, \& D'haenen, 2006) or using tDCS in a Simon task (Schroeder et al., 2016; Zmigrod, Zmigrod, \& Hommel, 2016).

It has been argued that response-time distribution analyses provide behavioral measures of response capture and response inhibition that are more process pure and more sensitive to experimental manipulations as well as individual differences compared to overall interference effects (for review, see van den Wildenberg et al., 2010). Here, we propose that such measures may also be more sensitive to the effects of neurostimulation, and by way of proof of concept we report the results of an rTMS study that endorse this claim. Before presenting this study, we briefly elaborate on the relevant literature on the conflict paradigm and the associated response-time distribution analyses.

\section{1 | The conflict paradigm}

Conflict paradigms provide informative measures of action control to resolve conflict situations that are characterized by the simultaneous activation of multiple response tendencies (Eriksen \& Eriksen, 1974; Lu \& Proctor, 1995). In the exemplary Simon task (Simon \& Rudell, 1967), participants are instructed to issue a fast left- or right-hand response to the color of a signal occurring to the left or right of visual fixation and to ignore the position of the stimulus on the screen. Although signal location is task irrelevant, responses are slower and more error prone on conflict trials when the signal occurs in the hemifield opposite to the response side signaled by the signal color (e.g., a colored signal calling for a right-hand response occurring on the left side of the screen).

Dual-process models provide elaborate experimental and conceptual frameworks for studying action control during conflict (Kornblum, Hasbroucq, \& Osman, 1990; Ridderinkhof, 2002a). The dual-process activation suppression (DPAS) model (Ridderinkhof, 2002b) invokes distributional analyses to dissociate two critical and temporally distinct cognitive processes that are masked when analyzing mean Simon effects. These sophisticated statistical analyses of RT and accuracy (distributional analyses) assess the neurocognitive mechanisms involved in these processes, their temporal dynamics, and individual differences therein (for review, see van den Wildenberg et al., 2010). Response capture, reflected by the number of impulsive response errors, emerges from conflicting action impulses. In the Simon task, this conflict emerges from conflicting information of location and color. Secondly, selective suppression is engaged to ward off and resolve the interference from those incorrect action impulses (Ridderinkhof, 2002a; van den Wildenberg et al., 2010; van Campen, Keuken, van den Wildenberg, \& Ridderinkhof, 2014). Using distributional analyses, for slower responses a decreasing interference effect is typical for the Simon task and has been linked to selective suppression in healthy controls and patients (Ridderinkhof, Forstmann, Wylie, Burle, \& van den Wildenberg, 2011; Wylie, Ridderinkhof, Bashore, \& van den Wildenberg, 2010).

Over the years, neuroimaging work has revealed how individual variations in brain structure as well as function covary with differences in action conflict resolution. In two studies, those brain-related measures were found to correlate with two behavioral measures from distributional analyses within the Simon task paradigm (Forstmann, Jahfari et al., 2008; Forstmann, van den Wildenberg, \& Ridderinkhof, 2008). Participants prone to response capture (i.e., those committing many fast response errors on conflict trials) showed increased pre-SMA activation. This suggests involvement of pre-SMA in the automatic action selection in the Simon task. The proficiency of suppressing conflicting action tendencies, expressed in reduced interference effects (reaction time difference between conflict and nonconflict trials) for slower responses with distributional analyses, covaried with both activation and white matter connectivity of the right inferior frontal cortex (rIFC; Forstmann, Jahfari et al., 2008; Forstmann, van den Wildenberg, \& Ridderinkhof, 2008), suggesting a direct role for IFC in suppression of unwanted response tendencies. Although these results are of 
correlational nature, clear hypotheses of the role of these brain regions within the Simon task are presented by these experiments. We will now introduce a proof-of-principle study to examine whether the response time (RT) distributional measures that are process specific to the expression and suppression of response capture, respectively, are sensitive to the effects of rTMS over the rIFC and pre-SMA.

\section{2 | Proof-of-principle study}

Extending beyond the correlational nature of brain-behavior associations, here we apply rTMS over pre-SMA and rIFC to test whether these cortical regions are causally involved in the expression and suppression of impulsive action tendencies, respectively. rTMS is an established tool to interfere offline with brain function to identify the causal roles of specific brain regions within a network (Walsh \& Cowey, 2000). Previous research with rTMS has indicated the causal involvement of rIFC in selective stopping (Aron, Behrens, Smith, Frank, \& Poldrack, 2007; Chambers et al., 2006) and the pre-SMA in action selection, switching response and rule alternatives, or processing conflict (Mars et al., 2009; Nachev, Kennard, \& Husain, 2008; Neubert, Mars, Buch, Olivier, \& Rushworth, 2010; Rushworth, Hadland, Paus, \& Sipila, 2002; Taylor, Nobre, \& Rushworth, 2007).

With rTMS, we will test in a causal manner two straightforward hypotheses, based on DPAS model predictions and ensuing neuroimaging work (Forstmann, Jahfari et al., 2008; Forstmann, van den Wildenberg, \& Ridderinkhof, 2008). Within a repeated-sessions pre- and poststimulation design, either pre-SMA or IFC is stimulated. In this way, the two stimulated brain regions are expected to affect two different parameters of distributional analyses and therefore act at the same time as ideal control stimulation regions. We expect that rTMS over pre-SMA induces stronger response capture, yielding an increase in fast impulsive errors on conflict trials in a Simon task after stimulation. This is captured within the distributional analyses as an increase in fast errors on conflict trials after stimulation compared to prestimulation behavior. In addition, perturbation of rIFC function is expected to impair selective suppression of conflicting action tendencies. This suppression, expressed in decreasing interference effects for slower responses prior to rTMS, is hypothesized to be diminished or even eliminated entirely, as expressed in increasing interference effects for slower responses after rTMS of the rIFC.

\section{2 | METHOD}

\section{1 | Participants}

The present study included 12 participants (7 women, $M=26$ years, $S D=4.37$ ) with normal or corrected-tonormal vision. All participants were screened according to the international screening guidelines for TMS research (Rossi, Hallett, Rossini, \& Pascual-Leone, 2009) and provided written informed consent prior to participation. All procedures complied with national laws and institutional guidelines, and were approved by the local ethics committee (institutional review board). One other initially recruited participant was excluded from participation prior to the TMS experiment due to high TMS threshold (above $60 \%$ of maximum stimulator output).

\section{2 | Apparatus}

\subsection{1 | Task}

A two-color (green and blue) Simon task was presented on a computer screen (17-inch digital display). The computer screen was placed in front of the participant at a distance of approximately $90 \mathrm{~cm}$, and the stimuli appeared at eye level. Each trial started with a fixation cross $(0.5 \times 0.5 \mathrm{~cm})$ in the middle of a white screen, which disappeared at the end of the trial (maximum duration of 1,500 ms). A colored circle (2-cm diameter) appeared on either the right or left side of the fixation cross (after either 750, 1,000, or 1,250 ms) and disappeared when a response was made (maximum duration of circle presentation was $1,500 \mathrm{~ms}$ ). If the trial lasted more than $800 \mathrm{~ms}$, a reminder appeared to respond faster. Each trial combination of stimulus color and location was pseudorandomized for each session. Exact stimulus repetitions occurred as low as practically feasible, ranging between $15.5 \%$ and $17.7 \%$ of total trials across participants.

Participants were instructed to issue a left- or right-hand button press as quickly and accurately as possible according to the color of the circle. For instance, participants responded right to blue circles and left to green circles; this mapping was counterbalanced over participants. Because circles could appear left or right of fixation, the (task-irrelevant) stimulus location inadvertently triggered an involuntary action impulse of the ipsilateral hand; that is, circles presented to the right side activate the right hand response, irrespective of color (see Figure 1). Based on the correspondence between the location of the stimulus and the correct response hand on a given trial, action selection according to color is either facilitated or hampered by the involuntary action impulse that is triggered by the position of the circle. Responses are typically fast and accurate on corresponding (CR) trials, in which the relevant and irrelevant processing streams converge to the same hand (i.e., when a green circle requiring a left-hand response is presented to the left of fixation). Alternatively, on noncorresponding (NCR) trials, RT is delayed and accuracy levels are typically lower because the two processing streams activate conflicting response tendencies (e.g., 


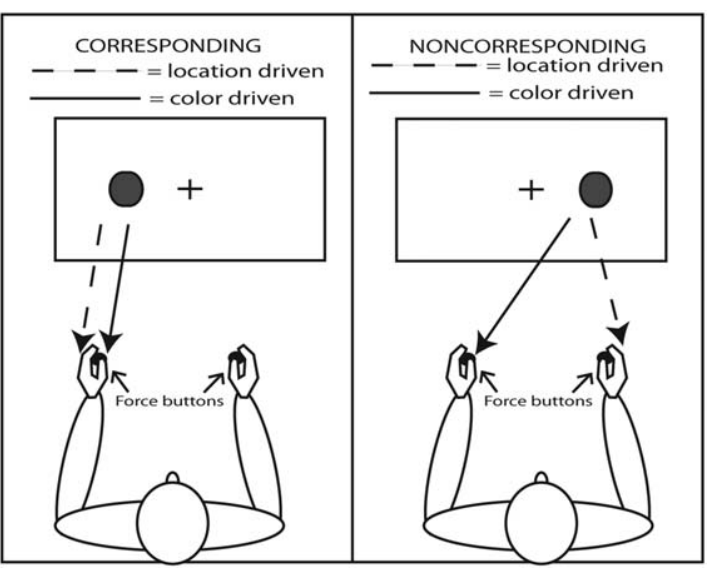

F I G URE 1 Simon task. Colored circles appear either on the left or right side of the screen. On corresponding (CR) trials, the location-driven and color-driven activations converge to the same hand. Location and color-driven activations are conflicting on noncorresponding (NCR) trials

when a green circle that should be responded to by the left hand is presented to the right of fixation).

\section{3 | Procedure}

Participants were familiarized with the task setup and the TMS setup during a behavioral session consisting of six experimental blocks of 96 trials each (not included in the analyses). The main goal of this first session was to introduce TMS and to show the participants what to expect within the experimental sessions. In this way, we hoped that any potential anxiety effects of participants for whom TMS was a new experience were reduced prior to the first experimental session. On different days (separated by at least 2 days), participants received either stimulation over rIFC or pre-SMA; the order was counterbalanced over participants. Before and after rTMS, the participants completed five experimental blocks of 96 trials (duration approximately 4 min per block) after performing 20 practice trials that were discarded. Participants received performance feedback after each experimental block.

\section{4 | rTMS}

Participants were comfortably seated in a chair during the entire experiment with a chin rest to minimize head movements. A 70-mm figure-of-eight coil was attached to the seat framework. Single-pulse TMS over the left and right motor cortex was conducted with a biphasic rapid 200 Magstim System to obtain the resting motor thresholds (RMT) for the abductor pollicis brevis (APB) muscle. Searching for the scalp location yielding the largest motor evoked potential (MEP) amplitude in the APB muscle started $2 \mathrm{~cm}$ lateral and $1 \mathrm{~cm}$ frontal to the vertex. RMT was measured, and the lowest intensity was taken at which the MEP amplitude was $>50 \mu \mathrm{V}$ in 5 out of 10 pulses. Overall, the mean RMT was 53\% of maximum stimulator output (ranging from $47 \%$ to 59\%). The stimulation intensity for both rTMS sessions was set at $90 \%$ of RMT of the left primary motor cortex. Participants received $1 \mathrm{~Hz}$ stimulation during $15 \mathrm{~min}$ (900 pulses in total) over the rIFC and pre-SMA on separate days. Both cortical areas were localized using a MRI-guided neuronavigation system (Visor; Advanced Neuro Technology, Enschede, The Netherlands). Individual MRI scans were acquired using a 3T Philips Achieva MRI system with a voxel size of $1 \mathrm{~mm} \times 1 \mathrm{~mm} \times 1 \mathrm{~mm}, 240 \mathrm{~mm}$ FOV. Averaged brain coordinates derived from a previous fMRI study with the same task (Forstmann, van den Wildenberg, \& Ridderinkhof, 2008) were translated into subject space, and the coil was placed directly above the targeted cortical region using neuronavigation. The rIFC (BA 44) was localized at $[x=38, y=20, z=4]$. The pre-SMA was localized at $[x=4$, $y=6, z=52$ ]. Coordinates are given in MNI (Montreal Neurological Institute) space. The coil was placed at an approximately 45-degree angle towards the midline. For the IFC, a similar configuration was used on the side of the head. Participants used foam earplugs during the experiment to reduce the noise of rTMS.

\section{5 | Data analysis}

\subsubsection{Overall mean RT and accuracy}

Overall mean RT and accuracy levels were calculated separately for $\mathrm{CR}$ and NCR trials. RTs longer than $2.5 S D$ were excluded on a subject-specific basis for each condition, resulting in the elimination of less than $7.3 \%$ of trials averaged over all conditions. Mean RT and accuracy data were submitted to repeated measures analysis of variance (ANOVA) using the within-subject factors TMS (pre-TMS vs. post-TMS), correspondence (CR vs. NCR), and area (rIFC vs. pre-SMA). These analyses were added to show how mean RT and accuracy conceal underlying patterns. In the following, the procedures for distributional analyses are outlined.

\subsection{2 | Hypothesis-driven distribution analyses}

To test our first hypothesis (rTMS over pre-SMA yields an increase in response capture), we focused on fast errors, graphically illustrated in conditional accuracy functions (CAFs). The measure for the strength of response capture, the proportion of fast errors, is revealed by CAFs that plot accuracy rates as a function of the entire Vincentized RT distribution for correct and incorrect responses together. For each correspondence level, RTs were rank ordered and 
partitioned into five equal-sized bins (quintiles; Segments 15). Accuracy rates were calculated for each segment, yielding five accuracy values each for CR and NCR trials, plotted against the average RT for each bin. The strength of impulse capture was analyzed by focusing on comparisons of the accuracy rates for the fastest RT segment of the CAFs on NCR trials.

For our second hypothesis (rTMS over rIFC will diminish the selective suppression of inappropriate action impulses), the focus is on the interference effect over time for the slowest responses, graphically represented in a delta plot. Delta plots quantified the temporal dynamics of interference control, plotting the Simon effect (i.e., mean RT[NCR] minus mean RT[CR]) as a function of RT. Delta plots involve rank ordering correct RTs for each level of correspondence, partitioning these values into five segments, and calculating the mean RTs for each correspondence level in each segment. Thus, Simon effects were computed for each segment; a smaller Simon effect in the slower end of the distribution signifies greater proficiency in selective suppression of action impulses (Ridderinkhof, 2002b).

We test our hypotheses using repeated measures ANOVA, followed by planned comparisons that zoom in on the fastest bin (for CAFs) and on the slowest bin (for delta plots), in accordance with our hypotheses. When the sphericity assumption was violated, degrees of freedom were corrected using the Greenhouse-Geisser (GG) method. Uncorrected $d f$ s are reported for ease of reading. Follow-up hypotheses were tested with a paired $t$ test.

\section{3 | RESULTS}

The results are organized as follows. First, the analyses on overall mean RT and accuracy are presented. Next, we present distributional analyses on CAFs and on delta plots. Overall mean RT and accuracy correspond to the geometric average of the five bins; hence, to avoid redundancy, in the distributional analyses we will not reiterate the analysis outcomes that do not involve the factor bin.

\subsection{Overall mean RT and accuracy}

Mean RT was analyzed using a three-way ANOVA with the factors area, TMS, and correspondence. Overall, mean RT was longer on NCR trials compared to CR trials, confirming the overall Simon effect (329 vs. $311 \mathrm{~ms}$, main effect of correspondence, $F(1,11)=121.337, p<.001)$. The interference effect was similar for rIFC and pre-SMA sessions (respectively, 322 vs. $317 \mathrm{~ms}$, main effect of area, $F(1,11)=2.347$, $p=.154$ ) and for pre- and postsessions (321 vs. $319 \mathrm{~ms}$, main effect of TMS, $F(1,11)=1.140, p=.308)$. The threeway interaction was not significant (Area $x$ TMS $\times$
Correspondence, $F(1,11)=3.153, p=.103$; all other interactions, $F<1$ ).

For accuracy, ANOVA with the factors area, TMS, and correspondence revealed differences between CR and NCR trials, reflecting lower accuracy on NCR trials compared to CR trials, again showing the typical Simon effect (87.4\% vs. $93.6 \%$, main effect correspondence, $F(1,11)=27.460$, $p<.001)$. Accuracy did not differ between rIFC and preSMA sessions $(90 \%$ vs. $91 \%$, main effect of area, $F(1,11)=$ $1.139, p=.309)$ or between pre- and poststimulation $(91 \%$ vs. $90 \%$, main effect of TMS, $F(1,11)=1.740, p=.214)$. The three-way interaction (Area $\times$ TMS $\times$ Correspondence, $F(1,11)=1.168, p=.303$ ) was not significant (all other interactions, $F<1$ ).

\subsection{Hypothesis-driven analyses of conditional accuracy functions}

ANOVA with the factors bin, TMS, area, and correspondence revealed typical CAF patterns (see Figure 2). That is, accuracy increases from fast to slow NCR trials, whereas accuracy is high for CR trials across the RT distribution. Accuracy levels changed as a function of response latency $(83.8 \%, 88.5 \%, 91.9 \%, 94.1 \%$, and $94.5 \%$ main effect of bin, $F(4,44)=28.877, p<.001$, GG-corrected: $\chi^{2}=33.404$, $\varepsilon=.407)$. Importantly for the distributional analyses, the accuracy patterns as a function of RT differed by correspondence $($ Bin $\times$ Correspondence, $F(4,44)=54.933, p<.001$, GG-corrected: $\left.\chi^{2}=17.601, \varepsilon=.619\right)$.

According to our response capture hypothesis, the accuracy levels for NCR trials within the fastest bin were expected to be reduced after pre-SMA stimulation but not after rIFC stimulation. Overall accuracy was similar for the two stimulated brain areas (main effect of area, $F(1,11)=$ $1.180, p=.300$ ) and between sessions (main effect of TMS, $F(1,11)=1.679, p=.222$ ). Additional interaction effects involving bin were not significant, Area $\times$ TMS $\times$ Bin, $F(4$, $44)=1.238, p=.309$, TMS $\times$ Correspondence $\times$ Bin, $F(4$, $44)=1.760, p=.154($ all other $F \mathrm{~s}<1)$.

Based on a priori hypotheses derived from the DPAS model, decreased accuracy level for NCR trials within the fastest RT bin were expected after pre-SMA stimulation (the response capture hypothesis, reflected in gray rectangles, Figure 2). However, accuracy for fast NCR responses did not change after rTMS over pre-SMA, $t(11)=.789$, $p=.447$. Likewise, comparing pre- and post-rIFC stimulation revealed similar accuracy for fast responses, $t(11)=-.500, p=.627$. In sum, and contrary to our expectations with respect to response capture, rTMS over pre-SMA did not increase the susceptibility for making fast impulsive response errors. Thus, the response capture hypothesis was not confirmed. 


\section{gof 13 PSYCHOPHYSIOLOGY}

IFC

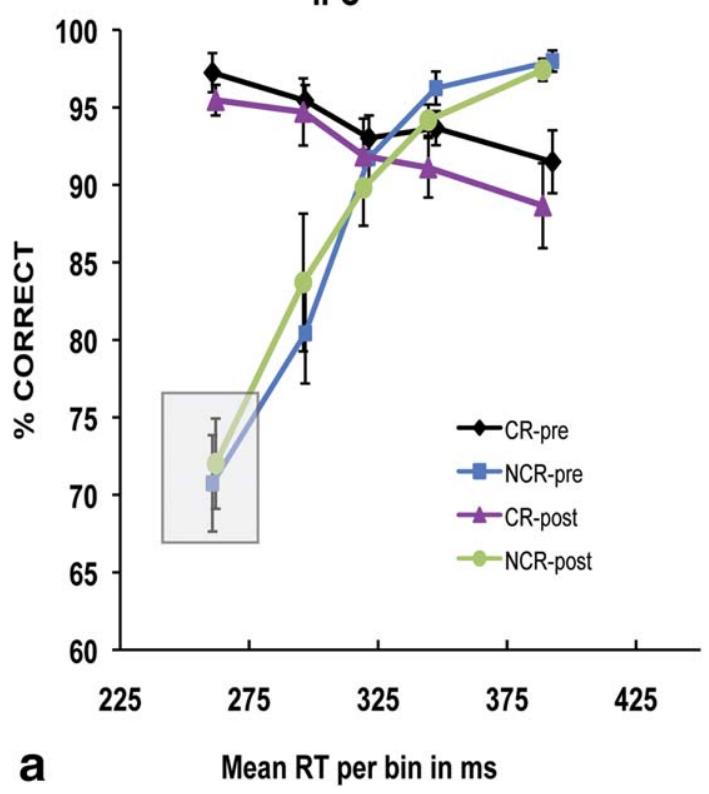

pre-SMA

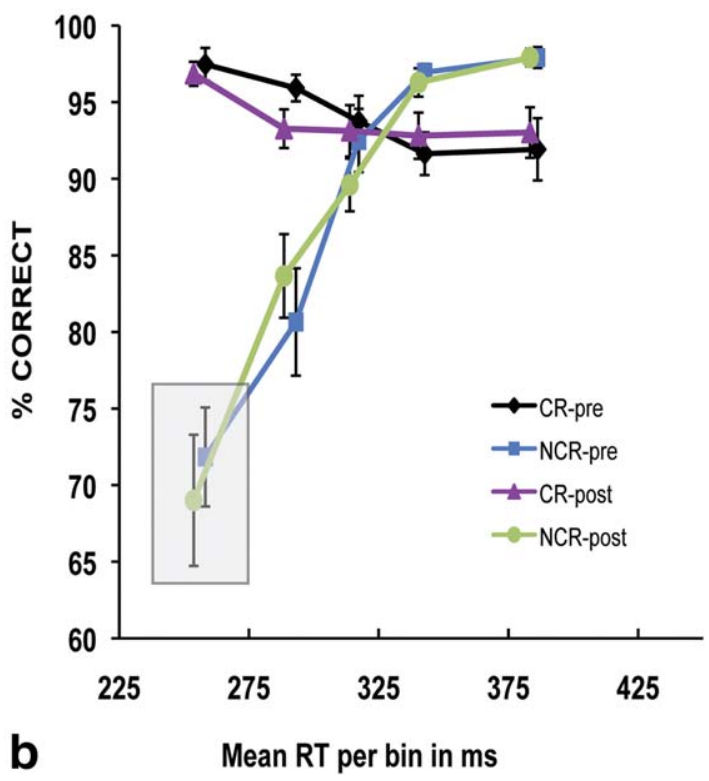

F I G URE 2 CAFs of pre- and post-rTMS over either rIFC or pre-SMA. To compute the conditional accuracy function (CAF), all reaction times for corresponding (CR) and noncorresponding (NCR) trials were rank ordered separately and then partitioned into five equal-sized bins representing the fastest to the slowest reactions. For each bin, an accuracy rate is calculated and plotted against the mean reaction time for that bin, creating a CAF spanning the entire distribution of reactions. The figure depicts the CAFs for CR (black and purple lines) and NCR (green and blue lines) trials related to (a) rIFC, and (b) pre-SMA stimulation. As expected, errors were predominantly associated with the fastest reactions (i.e., the fastest bin) on NCR trials, confirming that participants were susceptible to capture by the incorrect action impulses. rIFC and pre-SMA stimulation yielded similar patterns of fast errors, indicating that the strength of initial capture was equivalent. Gray rectangles represent planned comparisons. Error bars reflect standard error of the mean

\section{3 | Hypothesis-driven analyses of delta plots}

To quantify the temporal dynamics of interference control, we analyzed the interference effect (i.e., delta values) as a function of RT. Here, the delta values are taken as dependent measure (analyses of the slopes connecting the delta values yield similar results). The delta plots show the typical pattern: ANOVA with the factors bin, area, and TMS revealed changes in delta value for the different RT bins (see Figure 3 ) resulting in a decreased interference effect for longer RT $(22,26,26,25$, and $21 \mathrm{~ms}$, main effect of bin, $F(4,44)=$ 4.658, $p=.039$, GG-corrected: $\chi^{2}=40.478, \varepsilon=.331$ ).

This interference pattern differed between pre- and postsessions $($ Bin $\times$ TMS, $F(4,44)=14.418, p<.001$, GG-corrected: $\left.\chi^{2}=23.815, \varepsilon=.524\right)$. For the selective suppression hypothesis, the RT interference effect of the slowest bin was expected to increase after rIFC stimulation. Providing some support for this hypothesis, a trend was found for the interaction effect between bin, area, and TMS, suggesting differential temporal dynamics of interference control between preand postsessions for rIFC and pre-SMA (Bin $\times$ TMS $\times$ Area, $\quad F(4, \quad 44)=3.131, \quad p=.069, \quad$ GG-corrected: $\left.\chi^{2}=28.665, \varepsilon=.463\right)$. No significant other interaction effects involving the factor bin were obtained (all $F \mathrm{~s}<1$ ). The reduced RT interference effect for slower responses was diminished after rIFC stimulation, as expected based on specific a priori hypotheses (the selective suppression hypothesis, reflected in gray rectangles, Figure 3a). Indeed, the RT interference effect, when tested separately for the slowest RT bin, was significantly increased after rIFC stimulation (pre-rIFC stimulation $13 \mathrm{~ms}$ vs. post-IFC stimulation $30 \mathrm{~ms}, t(11)=4.351, p=.001)$. Pre-SMA stimulation did not affect interference control, $t(11)=.919, p=.378$.

\section{4 $\mid$ Summary of results}

To summarize, rIFC stimulation increased the RT interference effect for slower responses, thus confirming the selective suppression hypothesis. No latency effects of rTMS were observed after pre-SMA stimulation, indicating the causal involvement of the rIFC in suppressing action impulses. The expectation that pre-SMA stimulation should increase response capture (the response capture hypothesis: increased number of fast errors on conflict trials after stimulation) was not confirmed; rTMS over rIFC and pre-SMA had no effect on fast impulsive errors.

Trials following a CR trial typically show a larger interference effect compared to trials following a NCR trial (Egner, 2008). To check if sequential effects contributed to the findings reported above, the data were analyzed taking into account the correspondence of the previous trial. rTMS is expected to affect CAFs and delta plots, irrespective of the 

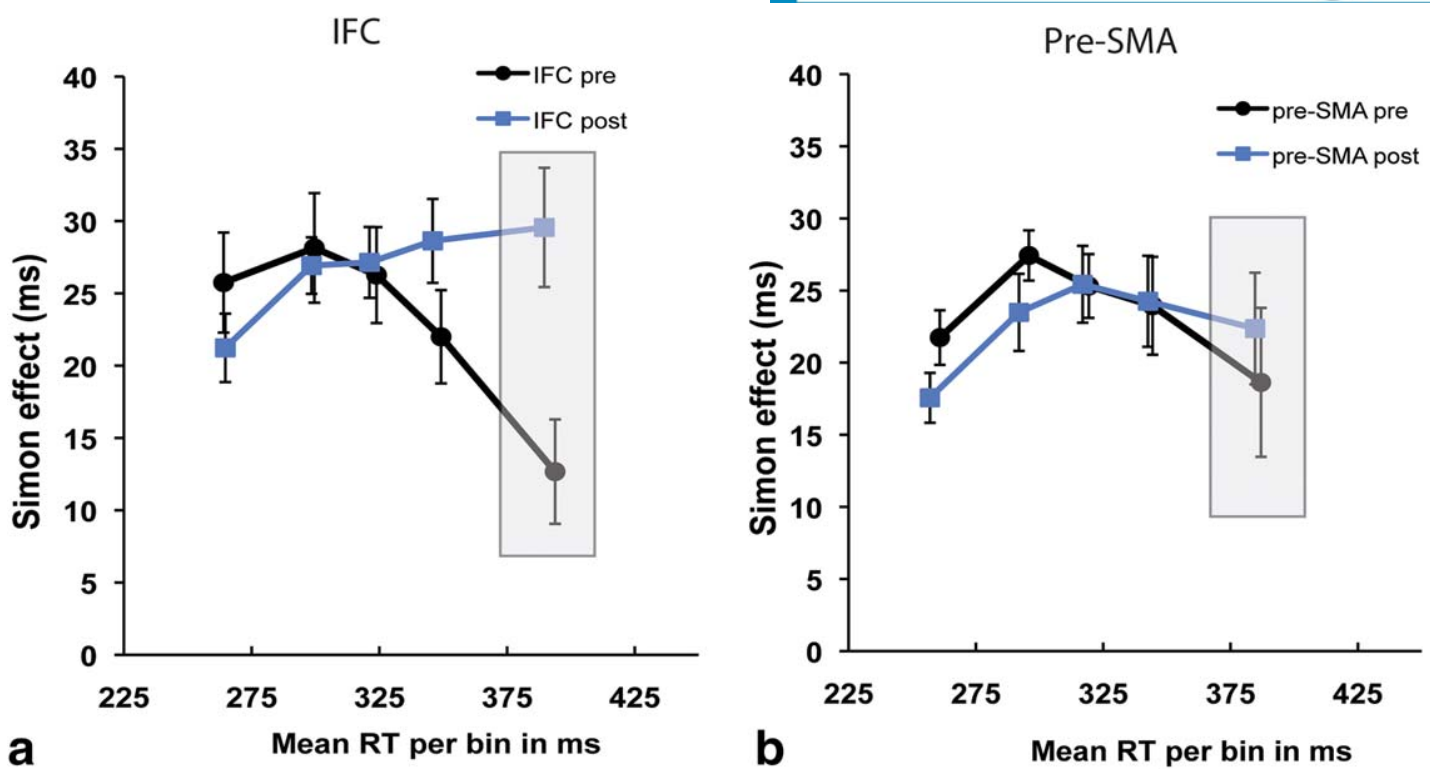

F I G URE 3 Delta plot of pre- and post-rTMS over either rIFC or pre-SMA. Delta plots show the interference effect over time. To compute a delta plot, reaction times (RT) for correct responses to corresponding (CR) and noncorresponding (NCR) trials were rank ordered separately and then partitioned into five equal-sized bins representing the fastest to the slowest reactions. For each bin, an interference effect was computed (mean RT for NCR trials minus mean RT for CR trials) and plotted against the mean RT for that bin. Black lines represent the delta plot for prestimulation sessions and blue lines represent the poststimulation sessions for (a) rIFC, and (b) pre-SMA. Gray rectangles represent the planned comparisons. Stimulation over rIFC resulted in increased $\mathrm{RT}$ interference effect for the slow responses, and this increase is absent after pre-SMA stimulation. Error bars reflect standard error of the mean

correspondence on the preceding trial. The results of these analyses are reported in the Appendix.

\section{4 | DISCUSSION}

In this study, we used rTMS to test the causal involvement of pre-SMA and rIFC on the expression and suppression of action impulses shown with distributional analyses. A priori hypotheses, derived from relevant neuroimaging research (Forstmann, Jahfari et al., 2008; Forstmann, van den Wildenberg, \& Ridderinkhof, 2008), hold that stimulation of preSMA should result in an increased proportion of fast impulsive errors in face of conflict (the response capture hypothesis). Stimulation of rIFC was expected to increase RT interference effects reflecting less suppression of unintended action impulses (the selective suppression hypothesis). The first hypothesis concerning the role of pre-SMA in response capture was not supported. No effect of stimulation was found on the accuracy level of fast responses (even when sequential effects were taken into account; see Appendix). The second hypothesis concerning the causal role of rIFC in selective suppression of action impulses, evidenced by a decreased interference effect for slower responses, was confirmed. The specificity of the effects seen after rIFC stimulation lends credibility to the null finding obtained for preSMA stimulation, even if a few caveats might apply. The importance of these findings is highlighted below, before returning to the issue of process-pure and sensitive measures in the study of neurostimulation effects on cognitive function.

\subsection{Expression of action impulses}

Pre-SMA has been suggested widely to play an important role in action selection (Mars et al., 2009; Nachev et al., 2008). This, together with the brain correlates reported by Forstmann, Jahfari et al., 2008; Forstmann, van den Wildenberg, and Ridderinkhof, 2008, led to the hypothesis that 1 Hz rTMS over pre-SMA would hamper response selection reflected by reduced accuracy for fast responses. However, pre-SMA stimulation did not affect accuracy, even though Figure $2 \mathrm{~b}$ seems to suggest a trend in the expected direction. The underlying reasons for this null finding could pertain to methodological or physiological factors, or simply to being underpowered.

First, the coordinates of the pre-SMA target obtained with fMRI were located deeper in the cortex than the coordinates of the rIFC. The distance between the TMS coil and the target might have reduced the efficiency and the impact of rTMS (Ruohonen \& Ilmoniemi, 2002; Stokes et al., 2005). The current coil orientation might be less efficient for pre-SMA stimulation, or the sample size may have been too low to detect a difference in behavior. Secondly, the preSMA contains not only excitatory neurons, but also inhibitory interneurons, that may be differentially impacted by the inhibitory effects of rTMS (Funke \& Benali, 2011; 
Robertson, Theoret, \& Pascual-Leone, 2003; Zilles \& Amunts, 2012).

Although our analysis of fast errors was argued to render more process-pure results than overall accuracy, a still more sensitive measure might be obtained in future studies by examining partial errors. Electromyographic (EMG) recordings from the response effectors, providing single-trial data on covert partial EMG errors, may prove useful in studying shifts in initial activation of an incorrect response (Allain, Burle, Hasbroucq, \& Vidal, 2009). A recent study showed that tDCS over the pre-SMA failed to affect the incidence of partial errors, but did reduce the number of those partial errors that turned into full manifest errors (Spieser, van den Wildenberg, Hasbroucq, Ridderinkhof, \& Burle, 2015), suggesting a subtle role of preSMA not in preventing rapid action impulses, but in preventing such impulses from expressing in overt errors.

Even if no effects of rTMS over pre-SMA were observed, this condition was still crucial, since it is mandatory to demonstrate that the effects of rIFC stimulation (discussed next) are unique to rIFC and do not generalize to stimulation of an active control site.

\subsection{Suppression of action impulses}

The key finding of this study is the evidence for the causal involvement of rIFC in selective suppression to counteract interference from involuntary action impulses, as evidenced by the rTMS-induced increased interference on the slow tail of the RT distribution. This finding provides a causal link to the previously reported relation between function and structure of the rIFC (Forstmann, van den Wildenberg, \& Ridderinkhof, 2008) and resembles similar findings in the domain of nonselective stopping (Chambers et al., 2006).

Note that a similar finding has also been reported outside the action control domain: rTMS of the rIFC resulted in impaired reasoning, resulting in enhanced belief-bias effects (Tsujii, Sakatani, Masuda, Akiyama, \& Watanabe, 2011). The model proposed by these authors entails a heuristic (fast and automatic) part and a logic-reasoning component (slow build up) not unlike the DPAS model used for present purposes, suggesting a more generic role of rIFC in suppression of immediate impulses beyond the motor system. The present findings add to that literature in that they demonstrate that the suppression of action impulses in conflict tasks, like the Simon task, critically relies on proper engagement of the rIFC.

The general role of the IFC in the suppression of action, as highlighted by a meta-analysis (Levy \& Wagner, 2011) and review (Aron, 2011), is extended here to the selective suppression of action impulses. On conflict trials in the Simon task, one action impulse is suppressed in favor of activation of the correct response alternative. Thus, the role of rIFC in such selective suppression may constitute a subprocess of a more generic class of situations characterized by the need to override actions. Previous work has pointed to a general role for IFC in action override, also in situations that focus less prominently on action suppression (Verbruggen, Aron, Stevens, \& Chambers, 2010). Future work should focus on the specific role of the IFC in action suppression and action override paradigms and their underlying neural implementation.

\subsection{Implications for neurostimulation studies}

Extensive review studies report that effects of noninvasive neurostimulation on cognitive functioning show results that lack in consistency and replicability. Beyond the many potential reasons for such a lack of robust findings that have been suggested previously, we have proposed that the lack of robustness may pertain to the dependent measures being too global and not sensitive enough to the demands of specific and subtle cognitive subprocesses. In conflict tasks, for instance, response interference effects were not modulated by stimulating the DLPFC using rTMS in a Stroop task (Vanderhasselt et al., 2006) or using tDCS in a Simon task (Schroeder et al., 2016; Zmigrod et al., 2016). In our lab, tDCS over the pre-SMA failed to produce any effects on overall interference measures in a spatial compatibility conflict task, but did show more subtle (but no less crucial) patterns when more fine-grained dependent measures were used (in this case, partial errors; Spieser et al., 2015).

Response-time distribution analyses have been argued to provide specific measures of response capture and response inhibition that are sensitive to experimental manipulations as well as clinical impairments, the effects of which remained obfuscated when limiting the analyses to overall interference effects (van den Wildenberg et al., 2010; Wylie et al., 2010). Here, we have demonstrated that such measures may also be more sensitive to the effects of neurostimulation.

Several limitations apply to this study, the most conspicuous of which is its modest sample size. Clearly, this study awaits replication at a larger one before the outcomes can be considered as robust. Also, the lack of a passive control condition leaves open the possibility of improvements due to test-retest practice, although the fact that improvement was observed after rIFC but not pre-SMA stimulation speaks against this possibility. At any rate, the results of this study suggest that the effects of neurostimulation may be subtle and concealed unless process-specific and sensitivedependent measures are used.

\subsection{Conclusion}

Overall, we conclude that rIFC stimulation impairs selective suppression, shown by an increased RT interference effect 
for slower responses, thus causally relating suppression of action impulses to rIFC function. No effects on fast impulsive errors were obtained after pre-SMA stimulation, leaving the exact role of the pre-SMA in conflict processing an open question.

\section{ACKNOWLEDGEMENTS}

This work was supported by an Open Competition grant (K. R. R., W. P. M. v. d. W.) from the Netherlands Organization for Scientific Research (NWO). Martijn Mulder and Birte Forstmann are gratefully acknowledged for making available MRI scans of selected participants for neuronavigation purposes.

\section{REFERENCES}

Allain, S., Burle, B., Hasbroucq, T., \& Vidal, F. (2009). Sequential adjustments before and after partial errors. Psychonomic Bulletin \& Review, 16(2), 356-362. https://doi.org/10.3758/PBR.16.2.356

Aron, A. R. (2011). From reactive to proactive and selective control: Developing a richer model for stopping inappropriate responses. Biological Psychiatry, 69(12), e55-e68. https://doi.org/10.1016/j. biopsych.2010.07.024

Aron, A. R., Behrens, T. E., Smith, S., Frank, M. J., \& Poldrack, R. A. (2007). Triangulating a cognitive control network using diffusion-weighted magnetic resonance imaging (MRI) and functional MRI. Journal of Neuroscience, 27(14), 3743-3752. https:// doi.org/10.1523/jneurosci.0519-07.2007

Chambers, C. D., Bellgrove, M. A., Stokes, M. G., Henderson, T. R., Garavan, H., Robertson, ... Mattingley, J. B. (2006). Executive "brake failure" following deactivation of human frontal lobe. Journal of Cognitive Neuroscience, 18(3), 444-455. https://doi. org/10.1162/089892906775990606

Duque, J., Olivier, E., \& Rushworth, M. (2013). Top-down inhibitory control exerted by the medial frontal cortex during action selection under conflict. Journal of Cognitive Neuroscience, 25(10), 1634-1648. https://doi.org/10.1162/jocn_a_00421

Egner, T. (2008). Multiple conflict-driven control mechanisms in the human brain. Trends in Cognitive Sciences, 12(10), 374-380. https://doi.org/10.1016/j.tics.2008.07.001

Eriksen, B. A., \& Eriksen, C. W. (1974). Effects of noise letters upon the identification of a target letter in a nonsearch task. Perception \& Psychophysics, 16(1), 143-149. https://doi.org/10.3758/ BF03203267

Forstmann, B. U., Jahfari, S., Scholte, H. S., Wolfensteller, U., van den Wildenberg, W. P. M., \& Ridderinkhof, K. R. (2008). Function and structure of the right inferior frontal cortex predict individual differences in response inhibition: A model-based approach. Journal of Neuroscience, 28(39), 9790-9796. https:// doi.org/10.1523/jneurosci.1465-08.2008

Forstmann, B. U., van den Wildenberg, W. P. M., \& Ridderinkhof, K. R. (2008). Neural mechanisms, temporal dynamics, and individual differences in interference control. Journal of Cognitive Neuroscience, 20(10), 1854-1865. https://doi.org/10.1162/jocn. 2008.20122
Funke, K., \& Benali, A. (2011). Modulation of cortical inhibition by rTMS-Findings obtained from animal models. Journal of Physiology, 589(18), 4423-4435. https://doi.org/10.1113/jphysiol.2011. 206573

Guse, B., Falkai, P., \& Wobrock, T. (2010). Cognitive effects of high-frequency repetitive transcranial magnetic stimulation: A systematic review. Journal of Neural Transmission, 117, 105-122. https://doi.org/10.1007/s00702-009-0333-7

Horvath, J. C., Forte, J. D., \& Carter O. (2015). Quantitative review finds no evidence of cognitive effects in healthy populations from single-session transcranial direct current stimulation (tDCS). Brain Stimulation, 8, 535-550. https://doi.org/10.1016/j.brs.2015.01.400

Hsu, W.-Y., Ku, X., Zanto, T. P., \& Gazzaley, A. (2015). Effects of noninvasive brain stimulation on cognitive function in healthy aging and Alzheimer's disease: A systematic review and metaanalysis. Neurobiology of Aging, 36, 2348-2359. https://doi.org/ 10.1016/j.neurobiolaging.2015.04.016

Jin, Y., Olk, B. \& Hilgetag, C. C. (2010). Contributions of human parietal and frontal cortices to attentional control during conflict resolution: A $1-\mathrm{Hz}$ offline rTMS study. Experimental Brain Research, 205, 131-138. https://doi.org/10.1007/s00221-010-2336-x

Kornblum, S., Hasbroucq, T., \& Osman, A. (1990). Dimensional overlap: Cognitive basis for stimulus-response compatibility: A model and taxonomy. Psychological Review, 97(2), 253-270. http://dx. https://doi.org/10.1037/0033-295X.97.2.253

Levy, B. J., \& Wagner, A. D. (2011). Cognitive control and right ventrolateral prefrontal cortex: Reflexive reorienting, motor inhibition, and action updating. Annals of the New York Academy of Sciences, 1224, 40-62. https://doi.org/10.1111/j.1749-6632.2011. 05958.x

Lu, C.-H., \& Proctor, R. W. (1995). The influence of irrelevant location information on performance: A review of the Simon and spatial Stroop effects. Psychonomic Bulletin \& Review, 2(2), 174207. https://doi.org/10.3758/BF03210959

Mars, R. B., Klein, M. C., Neubert, F.-X., Olivier, E., Buch, E. R., Boorman, E. D., \& Rushworth, M. F. S. (2009). Short-latency influence of medial frontal cortex on primary motor cortex during action selection under conflict. Journal of Neuroscience, 29(21), 6926-6931. https://doi.org/10.1523/jneuroci.1396-09.2009

Nachev, P., Kennard, C., \& Husain, M. (2008). Functional role of the supplementary and pre-supplementary motor areas. Nature Reviews Neuroscience, 9(11), 856-869. https://doi.org/10.1038/ $\operatorname{nrn} 2478$

Neubert, F.-X., Mars, R. B., Buch, E. R., Olivier, E., \& Rushworth, M. F. S. (2010). Cortical and subcortical interactions during action reprogramming and their related white matter pathways. Proceedings of the National Academy of Sciences of the United States of America, 107(30), 13240-13245. https://doi.org/10.1073/ pnas. 1000674107

Reteig, L. C., Talsma, L. J., van Schouwenburg, M. R., \& Slagter, H. A. (2017). Transcranial electrical stimulation as a tool to enhance attention. Journal of Cognitive Enhancement, 1, 10-25. https:// doi.org/10.1007/s41465-017-0010-y.

Ridderinkhof, K. R. (2002a). Micro- and macro-adjustments of task set: Activation and suppression in conflict tasks. Psychological Research, 66(4), 312-323. https://doi.org/10.1007/s00426-002-0104-7 
Ridderinkhof, K. R. (2002b). Activation and suppression in conflict tasks: Empirical clarification through distributional analyses. In W. Prinz \& B. Hommel (Eds.), Common mechanisms in perception and action. Attention \& performance (Vol. XIX, pp. 494519). Oxford, UK: Oxford University Press.

Ridderinkhof, K. R., Forstmann, B. U., Wylie, S. A., Burle, B., \& van den Wildenberg, W. P. M. (2011). Neurocognitive mechanisms of action control: Resisting the call of the sirens. Cognitive Science, 2(2), 174-192. https://doi.org/10.1002/wcs.99

Robertson, E. M., Theoret, H., \& Pascual-Leone, A. (2003). Studies in cognition: The problems solved and created by transcranial magnetic stimulation. Journal of Cognitive Neuroscience, 15(7), 948-960. https://doi.org/10.1162/089892903770007344

Rossi, S., Hallett, M., Rossini, P. M., Pascual-Leone, A., \& Safety of TMS Consensus Group. (2009). Safety, ethical considerations, and application guidelines for the use of transcranial magnetic stimulation in clinical practice and research. Clinical Neurophysiology, 120 (12), 2008-2039. https://doi.org/10.1016/j.clinph.2009.08.016

Ruohonen, J., \& Ilmoniemi, R. J. (2002). Physical principles for transcranial magnetic stimulation. A. Pascual-Leone, N. J. Davey, J. Rothwell, E. M. Wasserman, \& B. K. Puri (Eds.), Handbook of transcranial magnetic stimulation (pp. 18-30). New York, NY: Oxford University Press.

Rushworth, M., Hadland, K. A., Paus, T., \& Sipila, P. K. (2002). Role of the human medial frontal cortex in task switching: A combined fMRI and TMS study. Journal of Neurophysiology, 87 (5), 2577-2592. https://doi.org/10.1152/jn.00812.2001

Russowsky-Brunoni, A., \& Vanderhasselt, M. A. (2014). Working memory improvement with non-invasive brain stimulation of the dorsolateral prefrontal cortex: A systematic review and meta-analysis. Brain and Cognition, 86, 1-9. https://doi.org/10.1016/j. bandc.2014.01.008

Schroeder P. A., Pfister R., Kunde W., Nuerk H. C., \& Plewnia C. (2016). Counteracting implicit conflicts by electrical inhibition of the prefrontal cortex. Journal of Cognitive Neuroscience, 28, 1737-1748. https://doi.org/10.1162/jocn_a_01001

Simon, J. R., \& Rudell, A. P. (1967). Auditory SR compatibility: The effect of an irrelevant cue on information processing. Journal of Applied Psychology, 51(3), 300-304. https://doi.org/10.1037/h0020586

Spieser, L., van den Wildenberg, W. P. M., Hasbroucq, T., Ridderinkhof, K. R., \& Burle, B. (2015). Controlling your impulses: Electrical stimulation of the human supplementary motor complex prevents impulsive errors. Journal of Neuroscience, 35(7), 30103015. https://doi:10.1523/jneurosci.1642-14.2015

Stokes, M. G., Chambers, C. D., Gould, I. C., Henderson, T. R., Janko, N. E., Allen, N. B., \& Mattingley, J. B. (2005). Simple metric for scaling motor threshold based on scalp-cortex distance: Application to studies using transcranial magnetic stimulation. Journal of Neurophysiology, 94(6), 4520-4527. https://doi.org/10.1152/jn.00067.2005

Taylor, P. C. J., Nobre, A. C., \& Rushworth, M. F. S. (2007). Subsecond changes in top down control exerted by human medial frontal cortex during conflict and action selection: A combined transcranial magnetic stimulation electroencephalography study. Journal of Neuroscience, 27(42), 11343-11353. https://doi.org/10. 1523/jneurosciI.2877-07.2007

Tsujii, T., Sakatani, K., Masuda, S., Akiyama, T., \& Watanabe, S. (2011). Evaluating the roles of the inferior frontal gyrus and superior parietal lobule in deductive reasoning: An rTMS study. Neurolmage, 58(2), 640-646. https://doi.org/10.1016/j.neuroimage.2011.06.076

van Campen, A. D., Keuken, M. C., van den Wildenberg, W. P. M., \& Ridderinkhof, K. R. (2014). TMS over M1 reveals expression and selective suppression of conflicting action impulses. Journal of Cognitive Neuroscience, 26(1), 1-15. https://doi.org/10.1162/jocn_a_00482

van den Wildenberg, W. P. M., Wylie, S. A., Forstmann, B. U., Burle, B., Hasbroucq, T., \& Ridderinkhof, K. R. (2010). To head or to heed? Beyond the surface of selective action inhibition: A review. Frontiers in Human Neuroscience, 4. https://doi.org/10. 3389/fnhum.2010.00222

Vanderhasselt, M. A., De Raedt, R., Baeken, C., Leyman, L., \& D'haenen, H. (2006). The influence of rTMS over the left dorsolateral prefrontal cortex on Stroop task performance. Experimental Brain Research, 169, 279-282. https://doi.org/10.1007/s00221-005-0344-z

Verbruggen, F., Aron, A. R., Stevens, M. A., \& Chambers, C. D. (2010). Theta burst stimulation dissociates attention and action updating in human inferior frontal cortex. Proceedings of the National Academy of Sciences of the United States of America, 107(31), 13966-13971. https://doi.org/10.1073/pnas.1001957107

Walsh, V., \& Cowey, A. (2000). Transcranial magnetic stimulation and cognitive neuroscience. Nature Reviews Neuroscience, 1(1), 73-79. https://doi.org/10.1038/35036239

Wylie, S. A., Ridderinkhof, K. R., Bashore, T. R., \& van den Wildenberg, W. P. M. (2010). The effect of Parkinson's disease on the dynamics of online and proactive cognitive control during action selection. Journal of Cognitive Neuroscience, 22(9), 2058-2073. https://doi.org/10.1162/jocn.2009.21326

Zilles, K., \& Amunts, K. (2012). Segregation and wiring in the brain. Science, 335(6076), 1582-1584. https://doi.org/10.1126/science.1221366

Zmigrod, S., Zmigrod, L., \& Hommel, B. (2016). Transcranial direct current stimulation (tDCS) over the right dorsolateral prefrontal cortex affects stimulus conflict but not response conflict. Neuroscience, 322, 320-325. https://doi.org/10.1016/j.neuroscience.2016.02.046

How to cite this article: van Campen AD, Kunert R, van den Wildenberg WPM, Ridderinkhof KR. Repetitive transcranial magnetic stimulation over inferior frontal cortex impairs the suppression (but not expression) of action impulses during action conflict. Psychophysiology. 2018;55:e13003. https://doi.org/10.1111/ psyp. 13003

\section{APPENDIX}

\section{1 | EXPLORATORY SEQUENTIAL ANALYSES}

\section{1 | Sequential effects: Exploratory analyses}

Trials following a CR trial typically show a larger interference effect compared to trials following a NCR trial. 
Stimulation over rIFC is expected to result in reduced interference effect, irrespective of the correspondence on the preceding trial. Stimulation of pre-SMA with $1 \mathrm{~Hz}$ rTMS might increase the proportion of fast impulsive errors on NCR trials following a $\mathrm{CR}$ trial compared to following a conflict trial. To analyze these sequential effects, the extra factor sequence (preceding trial was $\mathrm{CR}$ vs. NCR) was added to the analyses presented in the main report. For ease of reading, only results that differ from those in the main report are mentioned. No clear hypothesis-driven effects were predicted; these analyses were conducted to verify that the main outcomes could not be attributed to an underlying sequential effect.

\section{2 | RESULTS}

\subsection{Conditional accuracy functions}

A five-way ANOVA, including the factor sequence, showed a similar general CAF pattern (main effect correspondence, $F$ $(1,11)=23.774, p<.001$, main effect bin, $F(4,44)=$ 30.459, $p<.001$, GG-corrected: $\chi^{2}=27.802, \varepsilon=.401$ ). According to the sequence effect, participants were indeed less accurate on trials following a CR trial (see Figure A1) compared to following a NCR trial $(90.1 \%$ vs. $91.5 \%$, main effect sequence, $F(1,11)=9.525, p=.010)$. The pattern of change over time for both $\mathrm{CR}$ and NCR trials depended on
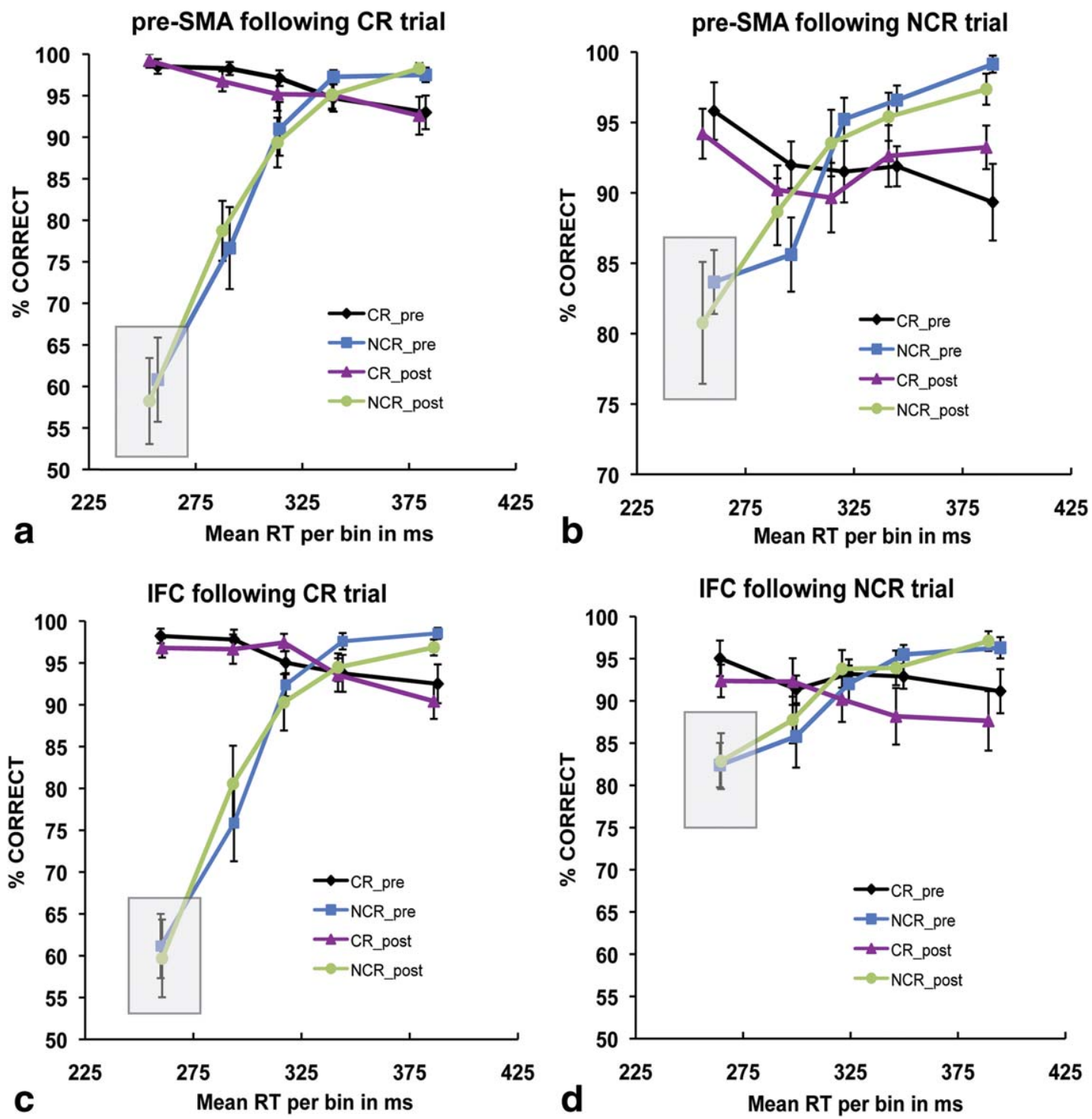

FIG URE A1 Sequential effects on accuracy for pre- and post-rTMS over either rIFC or pre-SMA. Accuracy percentages for NCR trials (blue and green lines) and CR trials (black and purple lines) are presented as a function of correspondence of the previous trial. Black and blue lines represent prerTMS, whereas green and purple lines represent post-rTMS sessions concerning (a) pre-SMA following CR, (b) pre-SMA following NCR, (c) rIFC following CR, and (d) rIFC following an NCR trial. As expected, errors were most frequent following CR trials compared to following NCR trials, confirming that participants were less susceptible to capture following conflict. Gray rectangles represent the planned comparisons. Error bars reflect standard error of the mean 
IFC

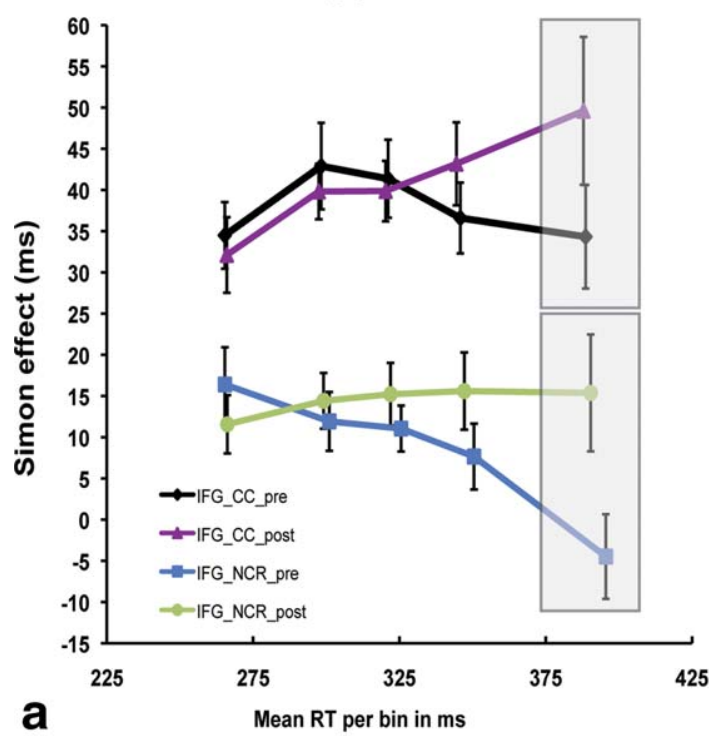

Pre-SMA

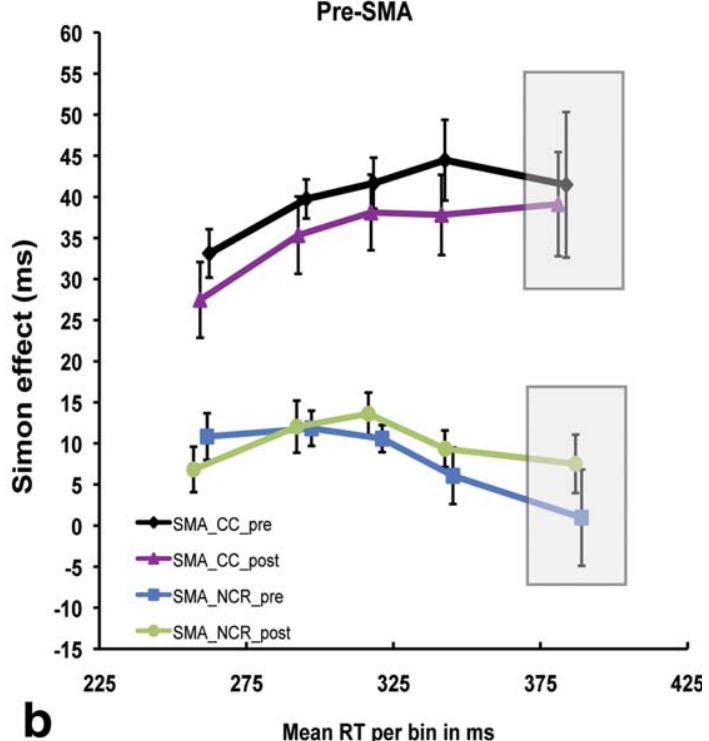

F IG URE A2 Delta plot showing sequential effects during pre- and post-rTMS over either rIFC or pre-SMA. Computation of delta plot is similar to description mentioned in Figure 3, except that the data were split according to the correspondence of the previous trial and rank ordered separately. The delta plot varied as a function of sequence, following a CR (black and purple lines) or NCR trial (blue and green lines). Black and blue lines represent the delta plot of the presessions, and the green and purple lines represent the delta plots from the postsessions for stimulation over (a) rIFC, and (b) pre-SMA. Gray rectangles represent the planned comparisons. Error bars reflect standard error of the mean

the correspondence level of the previous trial (interaction Sequence $\times$ Correspondence $\times$ Bin, $F(4,44)=20.631$, $p<.001$, GG-corrected: $\chi^{2}=21.025, \varepsilon=.650$, alongside interaction effects between sequence and correspondence, $F$ $(1,11)=43.803, p<.001$, sequence and bin, $F(4,44)=$ 20.313, $p<.001$, and between correspondence and bin, $F(4$, $44)=66.774, \quad p<.001, \quad$ GG-corrected: $\quad \chi^{2}=23.405$, $\varepsilon=.519)$. However, accuracy levels did not vary between pre- and post-TMS (main effect of TMS, $F(1,11)=1.695$, $p=.220$, all other $F \mathrm{~s}<1.5$ ).

Although the pertinent omnibus analyses of accuracy did not yield statistically reliable effects of rTMS, additional exploratory analyses were restricted to the fastest bin of the NCR CAF because, theoretically, response capture is most pronounced there. We focused on the same parameters as during the hypothesis-driven analyses. The planned comparisons (gray rectangles in Figure A1) on the first bin of the CAF revealed no differences in accuracy levels on NCR trials following a CR trial, $t$ $(11)=.477, p=.643$, or following a NCR trial, $t(11)=.813$, $p=.433$, in case of rTMS over pre-SMA, nor, for that matter, after rIFC stimulation (following CR trial, $t(11)=.437$, $p=.670$, or following NCR trial, $t(11)=-.161, p=.875)$.

\section{2 | Delta plots}

Four-way ANOVA with the factors bin, TMS, sequence, and area showed, first of all, an overall effect of sequence (39 vs. $10 \mathrm{~ms}$, main effect of sequence, $F(1,11)=93.915, p<.001)$. Secondly, the correspondence effect over time depended on the nature of the previous trial (interaction Sequence $\times$ Bin, $F$ $(4, \quad 44)=7.235, \quad p=.008, \quad$ GG-corrected: $\chi^{2}=36.939$, $\varepsilon=.388$ ). The delta plot (see Figure A2) is also modulated by TMS (interaction TMS $\times \operatorname{Bin}, F(4,44)=13.519, p<.001$, GG-corrected: $\left.\chi^{2}=34.213, \varepsilon=.440\right)$. Also, a differential pattern for the stimulated brain area was found (interaction effect, Area $\times$ TMS, $F(1,11)=6.286, p=.029)$. No clear overall changes over time for the delta value (bin, $F(4,44)=2.434$, $p=.134$, GG-corrected: $\left.\chi^{2}=42.018, \varepsilon=.335\right)$ or other interactions (Sequence $\times$ TMS, $F(1,11)=1.505, p=.245$; Area $\times$ TMS $\times$ Bin, $F(4,44)=2.689, p=.116$, GG-corrected: $\chi^{2}=42.139, \varepsilon=.329$, all other $F \mathrm{~s}<1$ ) were found.

Although a statistically reliable four-way interaction effect was absent, exploratory analyses were restricted to the slowest RT bin. Similar planned comparisons as used within the hypothesis-driven analyses (gray rectangles, see Figure A2) revealed that rTMS over rIFC caused an increased RT interference effect for slower responses for trials following a NCR trial, $t(11)=-3.249, p=.008$, but less so when the preceding trial was $\mathrm{CR}, t(11)=-1.848, p=.092$. No significant effects were found with respect to pre-SMA stimulation for both trials following a CR, $t(11)=.394, p=.701$, and following a NCR trial, $t(11)=-.975, p=.350$.

\section{3 | DISCUSSION}

To summarize, as anticipated, reliable sequential effects on both the delta plot and CAF were obtained. However, neither rTMS of pre-SMA nor IFC affected the percentage of fast 
impulsive errors. For the delta plot, rTMS over rIFC resulted in an increased RT interference effect for the slowest bin if the previous trial was a NCR trial. These effects were absent after pre-SMA stimulation.
These findings fit the typical patterns reported for sequential effects. Most important, they do not provide reason to suspect that the central outcomes presented in the main report could be attributed to underlying sequential effects. 